\title{
Time Warping of Apneic ECG Signals using Genetic Algorithm
}

\author{
Ga Ching Lui, Degang Wu, Ka Wai Cheung, Ho Fai Ma, Kwok Yip Szeto \\ Department of Physics \\ The Hong Kong University of Science and Technology \\ Hong Kong, China \\ Email: phszeto@ust.hk
}

\begin{abstract}
We construct a method of time warping in quasiperiodic time series analysis using genetic algorithm in order to extract the instantaneous phase difference between a template signal and a testing signal. Contrast to previous studies, which involves correlation estimations to determine the shape similarity of two signals taken from the quasiperiodic time series, time warping perform the comparison of the two signals by first constructing a discrete set of $M$ points formed from uniformly sampled values of the template signal $f(t)$. The discrete set of sample values of the testing signal, $g\left(t^{\prime}\right)$, which contains $N$ points, will be interpolated to form a continuous function so that the difference between the template signal at those $M$ points and the corresponding testing signals are minimize to best preserve the mapping of the two signals. The result of this optimization procedure produces a phase shift function that relates the time $\mathbf{t}^{\prime}$ in the testing signal to the time $t$ in the template signal. Due to the numerous choices in the partitioning of the time domain of the two signals, genetic algorithm is found to be effective in extracting this phase shift function. We apply this theoretical tool of time warping using genetic algorithm to analyze the electrocardiographic (ECG) signals, with the aim to investigate if central apneic and obstructive apneic episodes can be differentiated from non-apneic episodes. Detailed statistical analysis of the phase shift from real ECG data of sleep apnea patient indicates that the difference of both magnitude and phase of the signals can be used to differentiate apneic events from nonapneic events.
\end{abstract}

\section{INTRODUCTION}

The analysis of quasiperiodic time series is of great importance in a variety of fields such as biomedical science [1] $-[3]$, image processing [4], climatology [5] and finance [6], [7]. Frequency-domain algorithms have been extensively developed for the analysis of quasi-periodic signals, with wavelet transform [8] and Hilbert-Huang transform [9] being the prime examples that are frequently utilized by researchers. An alternative method is to perform the analysis of the time series in the time-domain. In this regard, Dynamic Time Warping (DTW) [10], for example, is a similarity measure [6], [11] which accommodates signals that are similar, but locally out of phase, by using non-linear alignments between two time series. This method has often been used as a similarity measure to classify time series [12], and is quite useful in the study of the relative phase relations between two time series similar in shape [4]. According to Syeda-Mahmood, Beymer and Wang [12], the ECG records of patients having the same type of cardiovascular diseases are often similar in shape, but often out of phase. Therefore DTW becomes an important tool for the study of sleep apnea. However, the performance of DTW depends on the many parameters that are often counterintuitively small and problem dependent [13], thereby limiting its use in practice. In order to overcome this limitation of DTW, we propose in this paper a genetic algorithm to perform the nonlinear alignment and we call this method time warping using genetic algorithm or TWGA.

The successful application of genetic algorithm (GA) has been demonstrated in fields such as biology [14]-[16] , clusters [17]-[19] and glass transition [20] in condensed matter physics. In engineering, genetic algorithm has also been used with good results in problems such as cyclicsteam oil production optimization problem [21], speed control of brushless DC motor [22], airport scheduling [23], mobile robot motion control [24], modeling adaptive agents in stock markets [25], [26], portfolio management [27] and traveling salesman problem [28]. GA has also been applied to optimize the parameters for DTW [29].

Different variants of DTW algorithms have been applied in the detection and classification of abnormal episodes in ECG records that may or may not belong to patients with cardiovascular diseases [12], [30]-[32], but mostly has one focused on the residual error [12], which is the difference in signal magnitude after alignment, and rarely has studied the phase shifts in ECG records. In this work we introduce a Time Warping Genetic Algorithm (TWGA), with application in the investigation of instantaneous phase differences between two electrocardiographic signal (ECG) signals for sleep apnea patients.

The time series of ECG signals consist of sequence of pulses. For an electrocardiographic signal (ECG), a quasiperiod, or a cardiac cycle, as shown in Fig 1, is comprised of a p-wave, a QRS complex and a t-wave corresponding to atrial depolarization, ventricular depolarization and ventricular repolarization respectively [12], [33].

As a demonstration, an example is given in Fig 2 showing the mapping function between two consecutive quasi-periods. In this example, the boundaries are defined to be the peak positions of the P-waves. The ECG data are obtained from MIT-BIH Polysomnographic Database [34] which can be accessed through http://physionet.org [35]. We mainly focus on the record slp60. Position of the P-waves can be extracted 


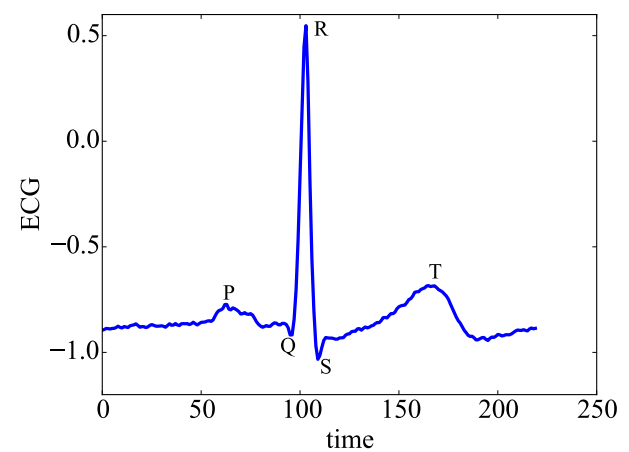

Fig. 1. An example of a quasi-period of ECG signal extracted from slp60 record . P and T denote the P-wave and T-wave. QRS denotes the QRScomplex.

with ecgpuwave from PhysioToolkit library [35]. The sampling rate for ECG is $250 \mathrm{~Hz}$ and the sleeping stage is recorded in the annotation files every 30 seconds. We will focus on the normal episodes(NE), central apneic (CA) and obstructive apneic (OA) episodes during sleep stage 1 of slp60.

The paper is organized into the several sections. We first define the problem of shape similarity measure in section II. We then introduce Time Warping Genetic Algorithm (TWGA) in section III and apply this method to ECG signals in section IV. The results along with detailed statistical analysis and hypothesis testing are in section V. We end the paper with a discussion of the possible application and future research of TWGA to medical time series as well as other fields in the last section.

\section{Problem Formulation: Shape Similarity}

In the application of classification, given two signals $F(t)$ and $G\left(t^{\prime}\right)$, where $t \in\left[0, T_{1}\right]$ and $t^{\prime} \in\left[0, T_{2}\right]$. The signals were rescaled so that the maximum and minimum are 1 and 0 :

$$
\begin{aligned}
& f(t)=\frac{F(t)-\min (F(t))}{\max (F(t))-\min (F(t))} \\
& g(t)=\frac{G(t)-\min (G(t))}{\max (G(t))-\min (G(t))}
\end{aligned}
$$

The variables of $t$ and $t^{\prime}$ are related by a function $\tau$ : $\left[0, T_{1}\right] \rightarrow\left[0, T_{2}\right]$ where $\tau$ is some function given by

$$
\tau=\underset{\tau^{\prime}(t)}{\arg \min } \int_{0}^{T_{1}} d t\left|f(t)-g\left(\tau^{\prime}(t)\right)\right| .
$$

In the analysis of real data, we consider a uniform sampling of the template signal $f(t)$ at $M+2$ points at $t_{i}, i=$ $0,1, \ldots, M+1$. The testing signal $g\left(t^{\prime}\right)$ is also uniformly sampled at $N+2$ points with equal spacing: $t_{j}, j=0,1, \ldots, N+1$. The discrete version of $\tau(t)$ is then defined by

$$
\left\{\tau_{i}\right\}=\underset{\left\{\tau_{i}^{\prime}\right\}}{\arg \min } \sum_{i=0}^{M+1}\left|f_{i}-\hat{g}_{i}\right|,
$$

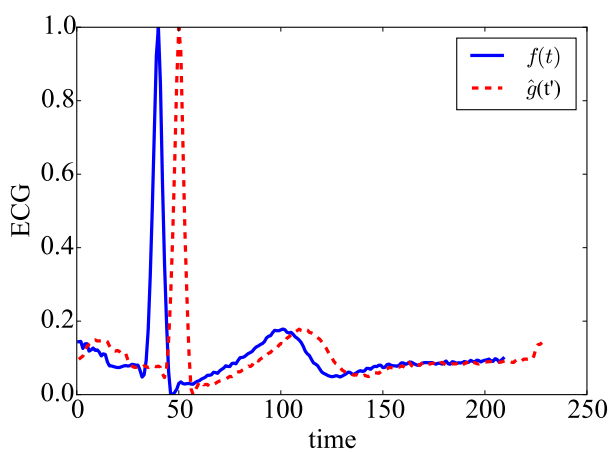

(a)

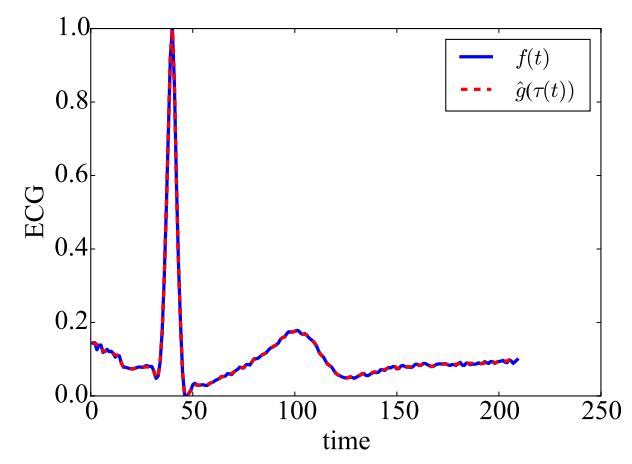

(b)

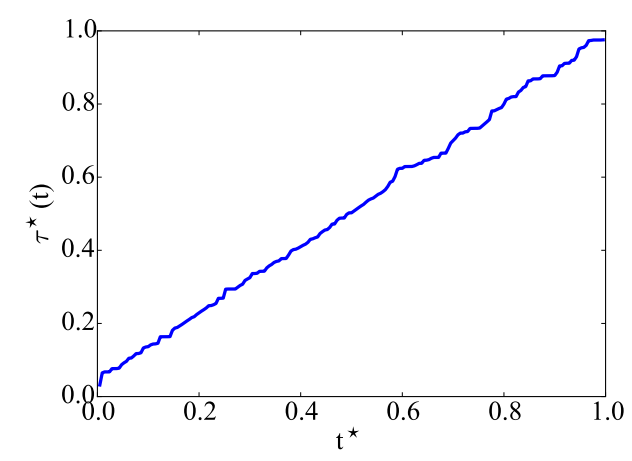

(c)

Fig. 2. An example of time warping to determine local transformation between reference signal and testing signal, which are two consecutive sections drawn from a time series of ECG signals in slp60 record. (a) The original ECG $f(t)$ is plotted with the another signal $g\left(t^{\prime}\right)$ before time warping. (b) The original ECG reference signal is plotted with testing signal after time warping. (c) The mapping $\tau^{\star}(t)$ is plotted against $t^{\star}$ where the $\star$ stands for normalization, see discussion in section IV

where $f_{i}=f\left(t_{i}\right)$ and $\hat{g}_{i}=\hat{g}\left(\tau_{i}^{\prime}\right)$ is the linear interpolation of $\left\{g_{j}\right\}$ where $g_{j}=g\left(t_{j}\right)$. The time mappings $\tau_{i}$ are defined to be the set of mappings $\tau_{i}^{\prime}$ that minimizes the absolute difference between sampled values of $f_{i}$ and interpolated values $\hat{g}_{i}$.

We illustrate the $\left\{\tau_{i}\right\}$ with an example. Consider $F(t)=$ $3 \sin (2 t)$ and $G\left(t^{\prime}\right)=\sin \left(t^{\prime}\right)$ in Fig 3 , such that $t \in[0, \pi]$ and $t^{\prime} \in[0,2 \pi]$. Suppose we choose $M=3$ so that the interval between each sampling for $f$ is $\Delta t=t_{i+1}-t_{i}=\pi / 4$. We then have $M+2=5$ samples are collected and normalized for $f$ : 


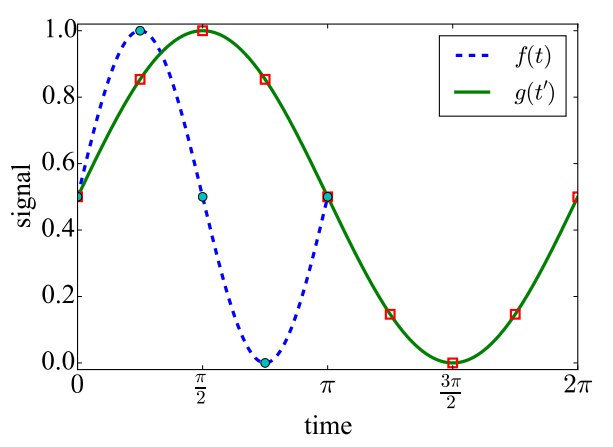

Fig. 3. An example illustrating the formulation of time mapping problem. The functions $f(t)$ and $g\left(t^{\prime}\right)$ are the normalized functions of $F(t)=3 \sin (2 t)$ and $G\left(t^{\prime}\right)=\sin \left(t^{\prime}\right)$ respectively. The blue dots and red square markers represent the sampled values obtained at fixed time intervals of $f(t)$ and $g\left(t^{\prime}\right)$ respectively.

$\left[f_{0}, f_{1}, f_{2}, f_{3}, f_{4}\right]=[0.5,1,0.5,0,0.5]$. Similarly, if we choose $\mathrm{N}=7$, we have $N+2=9$ samples for $g$. Note that $T_{1}$ and $T_{2}$ are $\pi$ and $2 \pi$. By (4), we obtain $\left\{\tau_{i}\right\}=\{0, \pi / 2, \pi, 3 \pi / 2,2 \pi\}$ for $i=0,1,2,3,4$, thus recovering the time mapping to be a uniform scaling by a factor of 2 . We will use this definition for time warping to analyze the pulses in ECG time series.

\section{Time Warping Genetic Algorithm}

Genetic algorithm (GA) is a heuristic searching method, inspired by natural selection in evolutionary theory. It is quite useful for optimization problems that involve a large solution space. A population of $N_{p}$ proposed solutions defined by different parameters is introduced, with each proposed solution defined as a chromosome with the parameters as encoded genes. These genes consist of loci and the phenotypes are expressed through the fitness function, which is the objective function of the optimization problem. Initially, a population of chromosomes is generated randomly and evolution of this population is driven mainly by elitism, which keeps the fittest individual chromosomes to the next generation. The change in the gene pool is generated through crossover and mutation. Crossover is used for exploration in the search for higher fitness and mutation is responsible for exploitation. In this section, we will introduce a type of GA that preserves the order of loci in the chromosome while maximizing the fitness function.

\section{A. Solution Representation}

Usually GA employs binary number to represent a solution. However, for optimization with real numbers, such as our time warping problem, the use of real numbers to represent solutions will be more efficient. In our work, every chromosome has $M$ loci, and each locus is a real number $\tau_{i}$. To initialize a chromosome, we generate $M$ random floating points numbers between 0 and $T_{2}$ with uniform distribution. Then the numbers are reordered in ascending order. The initial population has $N_{p}$ chromosomes. Using the previous example where $F(t)=3 \sin (2 t)$ and $G(t)=\sin (t)$, every

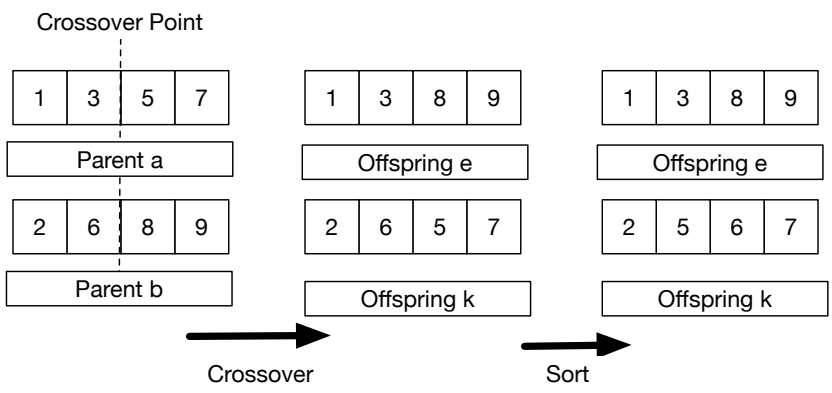

Fig. 4. An example of crossover operation at crossover point $\mathrm{c}=3$ involving single point crossover and sorting

chromosome will have a length of $3:\left[\lambda_{1}, \lambda_{2}, \lambda_{3}\right]$, where $T_{2}=2 \pi \geq \lambda_{3} \geq \lambda_{2} \geq \lambda_{1} \geq 0$.

\section{B. Fitness Function}

The fitness function of a chromosome $l$ is defined to be the inverse of the summation of the absolute difference between $f\left(t_{i}\right)$ and $g\left(\tau_{i}\right)$ :

$$
F_{l}=\frac{1}{\sum_{i=1}^{M}\left|f\left(t_{i}\right)-\hat{g}_{l}\left(\tau_{i}\right)\right|^{2}}
$$

Other metrics, such as absolute norm, can also be used. By maximizing $F_{l}$ we obtain the time mappings between two functions $f$ and $g$ which satisfy shape similarity in (4). For our example involving the sine functions, the value of fitness for chromosome $l$ can be calculated by $F_{l}=$ $1 /\left(\left|f\left(t_{1}\right)-\hat{g}\left(\lambda_{1}\right)\right|+\left|f\left(t_{2}\right)-\hat{g}\left(\lambda_{2}\right)\right|+\left|f\left(t_{3}\right)-\hat{g}\left(\lambda_{3}\right)\right|\right)=$ $1 /\left(\left|1-\hat{g}\left(\lambda_{1}\right)\right|+\left|0.5-\hat{g}\left(\lambda_{2}\right)\right|+\left|0-\hat{g}\left(\lambda_{3}\right)\right|\right)$.

\section{Parent Selection}

Based on the idea of natural selection, chromosomes with higher fitness would have a higher probability to pass down their genetic material to the next generation. In our algorithm, every chromosome in the population is ranked so that $F_{i} \geq F_{j}$ for $i \leq j$. The parents are selected from the fittest $N_{p}-N_{r}$ chromosomes to produce $N_{r}$ offspring chromosomes which replace chromosomes with low fitness. For the selection of each parent, the probability $P_{l}$ for chromosome $l$ to be chosen is:

$$
P_{l}=\frac{F_{l}}{\sum_{i=1}^{N_{p}-N_{r}} F_{i}}
$$

\section{Crossover Operation}

We use single-point crossover operation in this work. Suppose the two parent chromosomes candidates are $\left[a_{i}\right]$ and $\left[b_{i}\right]$ and the two offspring chromosomes are denoted by $\left[e_{i}\right]$ and $\left[k_{i}\right]$, where $i=1,2, \ldots, M$. The crossover point $1 \leq c \leq M$ is randomly chosen with equal probability $1 / M$. Then for the first offspring, we have $e_{i}=a_{i}$ for $i<c$ and $e_{i}=b_{i}$ for $i \geq c$; for the second offspring, $k_{i}=b_{i}$ for $i<c$ and $k_{i}=a_{i}$ for $i \geq c$. The loci in both offspring chromosomes will then 


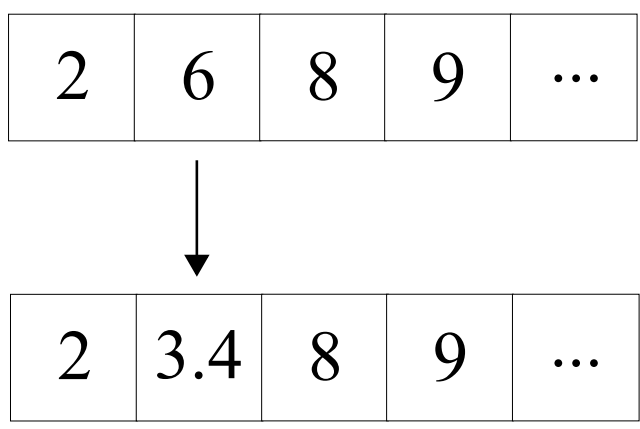

Fig. 5. An example of mutation operation at mutation point $i=2$. For mutation, the changed value is bounded so that $l_{3}=8 \geq l_{2} \geq l_{1}=2$.

be sorted and reordered in ascending order to make sure that $e_{i} \leq e_{j}$ and $k_{i} \leq k_{j}$ whenever $i<j$. An example is given in Fig 4 . The offspring chromosomes produced then replace the weakest $N_{r}$ chromosomes in the population.

\section{E. Mutation Operation}

In our algorithm, the chromosome with largest fitness value in the population will not be mutated. In chromosome $l$, the locus $l_{i}$ has a probability $P_{m}$ to mutate. The locus to be mutated will take a different value $x$ between the values at $l_{i-1}$ and $l_{i+1}$ with uniform probability. If $i=1$, the lower bound would be set to zero instead since that is the beginning position of a chromosome gene. Similarly, the upper bound of $l_{i}$ is $T_{2}$ if $i=M$. An example is given in Fig 5

\section{F. Iteration}

In each generation of the genetic algorithm, crossover operations and mutation operations will be performed on the population. The whole process is then iterated to search for the best solution. The iteration is terminated when a fixed number of generation is reached.

\section{ApPLICATION}

In this section, we will describe how to apply our proposed genetic algorithm on ECG signals of sleep apnea patients.

Again, the ECG data are obtained from MIT-BIH Polysomnographic Database [34] which can be accessed through http://physionet.org [35]. We mainly focus on the record $\operatorname{slp} 60$ in the database. Here, the start and end of a quasi-period are determined by consecutive positions of Rpeaks and positions of R-peaks are provided by the database. The sampling rate for ECG is $250 \mathrm{~Hz}$. The whole ECG time series is divided into 30 -sec segments, and every segment has an associated sleeping stage $(1,2,3,4$, REM or being awake), provided by the database. Also, every 30 -sec segment is labelled as normal episode (NE), central apneic (CA) and obstructive apneic (OA) episodes, etc. We will focus on the $\mathrm{NE}, \mathrm{CA}$ and OA episodes during sleep stage 1 of slp60. We denote the set of quasi-periods of ECG signals during NE (CA, or OA) episodes by $S_{N E}\left(S_{C A}, S_{O A}\right)$, respectively.

\section{A. Data preprocessing and preparation}

An ideal low-pass filter is applied with the cut-off frequency selected to be $(0.35)(250)=87.5 \mathrm{~Hz}$. In our analysis, $F(t)$ and $G\left(t^{\prime}\right)$ can be any signals from $S_{N E}, S_{C A}$ or $S_{O A}$. Before applying time warping, the signals $F(t)$ and $G\left(t^{\prime}\right)$ are rescaled so that the maximum and minimum of the rescaled voltage signals $f(t)$ and $g\left(t^{\prime}\right)$ are 1 and 0 .

\section{B. Time Warping}

The time mapping $\tau\left(t_{i}\right)$ is obtained using the methodology described in section III. Linear regression is carried out for $\tau\left(t_{i}\right)$ against $t_{i}$ to obtain the slope $m$ and $r^{2}$ where $r$ is the correlation coefficient. Both $\tau\left(t_{i}\right)$ and $t_{i}$ are then normalized to be $\tau^{\star}\left(t_{i}\right)$ and $t_{i}^{\star}$ so that the values range from 0 to 1 . The observable $q^{2}$, which is the linear deviation from straight line with unit slope, is defined as

$$
q^{2}=\frac{1}{M} \sum_{i=1}^{M}\left(\tau^{\star}\left(t_{i}\right)-t_{i}^{\star}\right)^{2},
$$

where $\mathrm{M}$ is the length of chromosome. The value of $\mathrm{M}$ depends on the number of sampled points in signal $F(t)$. For a quasiperiod in the ECG signal for $\operatorname{sip} 60, \mathrm{M}$ is around 200 since the sampling rate is $250 \mathrm{~Hz}$ and we are mapping all the sampled points, except for the $F\left(t_{0}\right)$ and $F\left(T_{1}\right)$, to $G\left(t^{\prime}\right)$. We group the time mappings $\tau\left(t_{i}\right)$ in the following fashion: Suppose $F(t)$ is drawn from set $S_{X}$ and $G\left(t^{\prime}\right)$ is drawn from set $S_{Y}$, where $\mathrm{X}$ and $\mathrm{Y}$ are $\mathrm{NE}, \mathrm{OA}$ or $\mathrm{CA}$, we denote the set of time mappings $\tau\left(t_{i}\right)$ between $F(t)$ and $G\left(t^{\prime}\right)$ to be $(\mathrm{X}-\mathrm{Y})$. Because both $X$ and $Y$ can be NE, OA or CA, we have nine sets of time mappings. Since sets of time mappings such as (NE-OA), (OA-NE) contain essentially the same information, we subtract three redundant sets of time mappings from the nine sets of time mappings to get six distinct sets of time mappings, consisting of the "intra-set" with three members: (NE-NE), (OA-OA) and (CA-CA) and the "inter-set" with three members: (NE-OA), (NE-CA) and (OA-CA).

As for the parameters in our TWGA, we set the population size in each generation to be $N_{p}=100$ and the number of chromosomes replaced in each generation to be $N_{r}=50$. The mutation probability for each locus is $P_{m}=0.02$ and the iteration is terminated at 1000th generation for the search of each time mapping. For each set of time mappings (XY), we randomly select one sample $F(t)$ from $S_{X}$ and one sample $G\left(t^{\prime}\right)$ from $S_{Y}$ and obtain the time mapping between $F(t)$ and $G\left(t^{\prime}\right)$ using TWGA. We then repeat this process for 1000 times to obtain a distribution of time mappings in (X$\mathrm{Y})$. Therefore, a distribution of $r^{2}$ and $q^{2}$ is collected for each set of time mappings (X-Y), such that $r^{2}$ is the coefficient of determination from linear regression of and $q^{2}$ is the linear deviation as defined in (7).

\section{Statistical Analysis}

We would like to investigate if there exist differences with statistical significance in the distribution of observables obtained from different sets of time mappings. Kruskal-Wallis 
$\mathrm{H}$ test [36] is a non-parametric statistical test used to determine if the population median of all groups are the same. The null hypothesis for Kruskal-Wallis Test, $H_{0}$, states that the samples of different groups are drawn from the same population by uniform sampling, whereas the alternative hypothesis, $H_{1}$, states that at least one of the groups have a different population median. Therefore, we can use the results of Kruskal-Wallis $\mathrm{H}$ test to reject $H_{0}$. If the p-value obtained from Kruskal-Wallis $\mathrm{H}$ test is less than 0.05 , we should reject $H_{0}$ and accept the alternative hypothesis $H_{1}$. If Kruskal-Wallis $\mathrm{H}$ test supports $H_{1}$, Mann-Whitney $\mathrm{U}$ test [37] is applied for pairwise post hoc testing to determine which of the groups are different from the rest of the population, as observed by Kruskal-Wallis $\mathrm{H}$ test. Since type I error, which is the false rejection of a null hypothesis, increases under this algorithm for multiple comparison, the critical p-value for Mann-Whitney $U$ test is modified by Bonferroni correction [38], which states that $\alpha$ should be divided by $C_{2}^{h}$, which is the number of possible pairs among the groups, if we are testing for $\mathrm{h}$ groups.

We denote the distribution of linear deviation $q^{2}$ from the time mappings in set $(\mathrm{X}-\mathrm{Y})$ by $P_{X-Y}\left(q^{2}\right)$. Similarly, the distribution of coefficient of determination $r^{2}$ from the time mappings in set $(\mathrm{X}-\mathrm{Y})$ is denoted by $P_{X-Y}\left(r^{2}\right)$. KruskalWallis H Test is applied on both types of distribution.

\section{RESUlts}

Signals $F(t)$ and $G\left(t^{\prime}\right)$ were drawn from the three sets of signals that occurs during sleep stage 1: normal $\left(S_{N E}\right)$, obstructive apnea $\left(S_{O A}\right)$ and central apnea $\left(S_{C A}\right)$. We are interested in obtaining the distributions of two variables: $P_{X-Y}\left(r^{2}\right)$ and $P_{X-Y}\left(q^{2}\right)$. The coefficient of determination $r^{2}$ from linear interpolation of the time mapping function is the simply the square of correlation coefficient. The symbol $q^{2}$ denotes linear deviation, define in (7) as deviation of the $\tau_{i}^{\star}$ value from $t_{i}^{\star}$.

Kruskal-Wallis test is applied on the distributions $P_{X-Y}\left(q^{2}\right)$. The results are tabulated in Table I which shows that Kruskal-Wallis H Test supports the alternative hypothesis $H_{1}$ for the distribution of linear deviation. It follows that for $P_{X-Y}\left(q^{2}\right)$ there is at least one group with a population median different from the rest of the groups with statistical significance at confidence level $\alpha=0.05$ as the P-value is less than $\alpha$. Alternatively, since the value of the test statistics, or the $\mathrm{H}$-value, is larger that the critical value $H^{c}=11.07$ corresponding to $\alpha=0.05$, the null hypothesis is rejected. Similarly, Kruskal-Wallis $\mathrm{H}$ Test is also applied on the distributions $P_{X-Y}\left(r^{2}\right)$, and the test supports the alternative hypothesis $H_{1}$ that for $P_{X-Y}\left(r^{2}\right)$ there is at least one group with a population median different from the rest of the groups with statistical significance at confidence level $\alpha=0.05$.

We therefore proceed to carry out post hoc test for both linear deviation $q^{2}$ and coefficient of determination $r^{2}$. Because we have 6 sets of time mappings corresponding to 6 explanatory variables, there are altogether fifteen cases for pairwise testing considering that the pair $\left(\mathrm{X}_{1}-\mathrm{Y}_{1}\right)$ and $\left(\mathrm{X}_{2}\right.$ -
TABLE I

RESUlts of KRUSKAL-WALlis H TEST RECORDing THE H-VALUE, F-VALUE AND THE CORRESPONDING CONFIDENCE P-VALUE

\begin{tabular}{|c||c|c|}
\hline & H-value & P-value \\
\hline$q^{2}$ & 25.64 & $1.046 \mathrm{e}-04$ \\
\hline$r^{2}$ & 21.89 & $5.476 \mathrm{e}-04$ \\
\hline
\end{tabular}

TABLE II

RESULTS OF MANN-WHITNEY U TEST ON AS POST HOC TEST FOR KRUSKAL-WALLIS H TEST ON COEFFICIENT OF DETERMINATION $q^{2}$

\begin{tabular}{|c||c|c||c|c|}
\hline Case & $(\mathrm{X}-\mathrm{Y})$ & $\left(\mathrm{X}^{\prime}-\mathrm{Y}^{\prime}\right)$ & U-value & P-value \\
\hline 1 & (NE-NE) & (OA-OA) & 464651.0 & $6.19 \mathrm{e}-03$ \\
\hline 2 & (NE-NE) & (CA-CA) & 488780.0 & $3.85 \mathrm{e}-01$ \\
\hline 3 & (NE-NE) & (NE-OA) & 482836.0 & $1.84 \mathrm{e}-01$ \\
\hline 4 & (NE-NE) & (NE-CA) & 482412.0 & $1.73 \mathrm{e}-01$ \\
\hline 5 & (NE-NE) & (CA-OA) & 483591.0 & $2.04 \mathrm{e}-01$ \\
\hline 6 & (OA-OA) & (CA-CA) & 453132.0 & $2.84 \mathrm{e}-04$ \\
\hline 7 & (OA-OA) & (NE-OA) & 447235.0 & $4.39 \mathrm{e}-05$ \\
\hline 8 & (OA-OA) & (NE-CA) & 446820.0 & $3.82 \mathrm{e}-05$ \\
\hline 9 & (OA-OA) & (CA-OA) & 448262.0 & $6.16 \mathrm{e}-05$ \\
\hline 10 & (CA-CA) & (NE-OA) & 494213.0 & $6.54 \mathrm{e}-01$ \\
\hline 11 & (CA-CA) & (NE-CA) & 494229.0 & $6.55 \mathrm{e}-01$ \\
\hline 12 & (CA-CA) & (CA-OA) & 495152.0 & $7.07 \mathrm{e}-01$ \\
\hline 13 & (NE-OA) & (CA-OA) & 499643.0 & $9.78 \mathrm{e}-01$ \\
\hline 14 & (NE-OA) & (NE-CA) & 499746.0 & $9.84 \mathrm{e}-01$ \\
\hline 15 & (NE-CA) & (CA-OA) & 499473.0 & $9.67 \mathrm{e}-01$ \\
\hline
\end{tabular}

$\left.Y_{2}\right)$ is the same as the pair $\left(X_{2}-Y_{2}\right)$ and $\left(X_{1}-Y_{1}\right)$. For each case, we compare (X-Y) with ( $\left.\mathrm{X}^{\prime}-\mathrm{Y}^{\prime}\right)$.

For $P_{X-Y}\left(q^{2}\right)$, since Kruskal-Wallis $\mathrm{H}$ test rejects $H_{0}$, we carry out Mann-Whitney U Test for further analysis following Kruskal-Wallis $\mathrm{H}$ test to find out which of the groups is responsible for the deviation from the entire population. The results are shown in Table II. The P-value has to be less than the adjusted value of $\alpha=0.0033$ by Bonferroni correction for statistical significant deviations. From the table, case 1: (NE-NE) with (OA-OA), case 6:(OA-OA) with (CA-CA), case 7:(OA-OA) with (NE-OA), case 8:(OA-OA) with (NE-CA) and case 9:(OA-OA) with (CA-OA) show statistically significant differences in sample medians. This indicates the phase shift between intra-set time mappings of signals extracted from OA episodes is different not only from that obtained in other intra-set time mappings but also from that obtained in all interset time mappings. Intra-set time mappings (X-X) including (NE-NE), (OA-OA) and (CA-CA) refers to comparing two signals drawn from the same set $S_{X}$; whereas inter-set time mappings (X-Y) such as (NE-OA), (NE-CA) and (OA-CA) refers to comparing a signal in set $S_{X}$ with another signal in a different set $S_{Y}$.

On the other hand, since Kruskal-Wallis $\mathrm{H}$ test also rejects $H_{0}$ for $P_{X-Y}\left(r^{2}\right)$, Mann-Whitney $\mathrm{U}$ Test is again carried out as a post hoc test. The results are shown in Table III Contrast to the analysis of $q^{2}$, case 1 shows no statistical dominance over the whole population. The distribution of $r^{2}$ 
TABLE III

RESUlTS OF MANN-WHITNEY U TEST ON AS POST HOC TEST FOR KRUSKAL-WALLIS H TEST ON COEFFICIENT OF DETERMINATION $r^{2}$

\begin{tabular}{|c||c|c||c|c|}
\hline Case & $(\mathrm{X}-\mathrm{Y})$ & $\left(\mathrm{X}^{\prime}-\mathrm{Y}^{\prime}\right)$ & U-value & P-value \\
\hline 1 & (NE-NE) & (OA-OA) & 483246.0 & $1.94 \mathrm{e}-01$ \\
\hline 2 & (NE-NE) & (CA-CA) & 475194.0 & $5.47 \mathrm{e}-02$ \\
\hline 3 & (NE-NE) & (NE-OA) & 469188.0 & $1.70 \mathrm{e}-02$ \\
\hline 4 & (NE-NE) & (NE-CA) & 475277.0 & $5.56 \mathrm{e}-02$ \\
\hline 5 & (NE-NE) & (CA-OA) & 473288.0 & $3.86 \mathrm{e}-02$ \\
\hline 6 & (OA-OA) & (CA-CA) & 457914.0 & $1.12 \mathrm{e}-03$ \\
\hline 7 & (OA-OA) & (NE-OA) & 452592.0 & $2.41 \mathrm{e}-04$ \\
\hline 8 & (OA-OA) & (NE-CA) & 457729.0 & $1.06 \mathrm{e}-03$ \\
\hline 9 & (OA-OA) & (CA-OA) & 456755.0 & $8.11 \mathrm{e}-04$ \\
\hline 10 & (CA-CA) & (NE-OA) & 493437.0 & $6.11 \mathrm{e}-01$ \\
\hline 11 & (CA-CA) & (NE-CA) & 499610.0 & $9.76 \mathrm{e}-01$ \\
\hline 12 & (CA-CA) & (CA-OA) & 497250.0 & $8.31 \mathrm{e}-01$ \\
\hline 13 & (NE-OA) & (CA-OA) & 494082.0 & $6.47 \mathrm{e}-01$ \\
\hline 14 & (NE-OA) & (NE-CA) & 495749.0 & $7.42 \mathrm{e}-01$ \\
\hline 15 & (NE-CA) & (CA-OA) & 498523.0 & $9.09 \mathrm{e}-01$ \\
\hline
\end{tabular}

during OA episodes shows no statistically significant deviation from that obtained during normal episodes. Nonetheless, all other cases involving (OA-OA) still shows deviations with statistical significance. Therefore, observable $r^{2}$ also captures the difference in phase distortion between signals obtained during OA periods and signals obtained for inter-set mappings.

\section{DISCUSSION}

In our short study, we have demonstrated the use of TWGA as a tool to study the phase difference. We then apply the algorithm on the slpdb60 ECG signals, and show that ECG signals obtained during different sleeping states exhibit different phase shifts as captured by linear deviation $q^{2}$ and coefficient of determination $r^{2}$ from linear interpolation. In particular, ECG signals obtained during OA episodes show statistically significant differences from signals obtained during CA episodes and during NE periods in terms of $r^{2}$ and $q^{2}$ related to phase distortion extracted by TWGA. This may facilitate the development of autonomous classification systems incorporating extra information regarding phase shifts that differentiates obstructive apneic ECG signals from both central apneic signals and normal signals with enhanced accuracies. Such systems may greatly reduce medical resources allocated for diagnosis of sleep apnea among other diseases. Further statistical study is required on an expanded database with more patients with different conditions for the purpose of classifying different apneic episodes and understanding the biological process in sleep apnea. Characteristics of quasiperiodic biological time-series that are universal among different patients can be extracted and these common signal features may provide insights in the mechanisms of sleep apnea and other diseases such as arrhythmia. Moreover, other measures of phase changes may be considered apart from $q^{2}$ and $r^{2}$ in order to better understand the instantaneous local changes in a quasi-period. This allows TWGA to be utilized as a tool to observe the variation of phase shift as a dynamic process. Furthermore, the information of local phase shifts obtained by TWGA can be useful in embedding of time-series in the phase space. A limitation of this algorithm involves the fluctuation of signal strength as mentioned in section V. A possible remedy would be to use some non-constant scaling functions to normalize signal $F$ and $G$ instead of using a fixed scale factor. Another approach is to study different components of one quasi-period (such as either p-wave, QRS complex or T-wave in an ECG signal) seperately [33]. Alternatively, we can use the difference of derivatives of the testing signal and reference signal instead of the difference in magnitude [39], [40]. Since this requires the first derivative of the functions to be continuous, linear interpolation described in section III should be replaced by cubic spline interpolation.

\section{CONCLUSION}

In this paper, we have developed a novel algorithm for shape matching across different signals in the time domain with genetic algorithm(TWGA). TWGA can be used to more accurately study the phase shift in a quasiperiodic function. In particular, we apply the algorithm on the ECG signals of a sleep apnea patient and collect statistical data for analysis. We show that the distribution of phase-related variables obtained during obstructive apneic epsodes (OA) is different from that obtained during central apneic (CA) and normal episodes (NE).

\section{ACKNOWLEDGMENT}

K. Y. Szeto acknowledges the support of grant FSGRF13SC25 and FSGRF14SC28.

\section{REFERENCES}

[1] C. Alexakis, H. Nyongesa, R. Saatchi, N. Harris, C. Davies, C. Emery, R. Ireland, and S. Heller, "Feature extraction and classification of electrocardiogram (ECG) signals related to hypoglycaemia." IEEE, 2003, pp. 537-540, conference Proceedings.

[2] P. Fonseca, X. Long, M. Radha, R. Haakma, R. M. Aarts, and J. Rolink, "Sleep stage classification with ECG and respiratory effort," Physiological Measurement, vol. 36, no. 10, p. 2027, 2015.

[3] E. Pereda, R. Q. Quiroga, and J. Bhattacharya, "Nonlinear multivariate analysis of neurophysiological signals," Progress in Neurobiology, vol. 77, no. 12, pp. 1-37, Sep. 2005. [Online]. Available: http: //www.sciencedirect.com/science/article/pii/S030100820500119X

[4] Y. Makihara, N. T. Trung, H. Nagahara, R. Sagawa, Y. Mukaigawa, and Y. Yagi, "Phase Registration of a Single Quasi-Periodic Signal Using Self Dynamic Time Warping," in Computer Vision ACCV 2010, ser. Lecture Notes in Computer Science, R. Kimmel, R. Klette, and A. Sugimoto, Eds. Springer Berlin Heidelberg, Nov. 2010, no. 6494 , pp. 667-678, dOI: 10.1007/978-3-642-19318-7_52. [Online]. Available: http://link.springer.com/chapter/10.1007/978-3-642-19318-7_52

[5] R. C. Stone, G. L. Hammer, and T. Marcussen, "Prediction of global rainfall probabilities using phases of the Southern Oscillation Index," Nature, vol. 384, no. 6606, pp. 252-255, Nov. 1996. [Online]. Available: http://www.nature.com/nature/journal/v384/n6606/abs/384252a0.html

[6] P. Tsinaslanidis, A. Alexandridis, A. Zapranis, and E. Livanis, "Dynamic Time Warping as a Similarity Measure: Applications in Finance," no. Journal Article, 2014.

[7] Y. Bodyanskiy and S. Popov, "Neural network approach to forecasting of quasiperiodic financial time series," European Journal of Operational Research, vol. 175, no. 3, pp. 1357-1366, Dec. 2006. [Online]. Available: http://www.sciencedirect.com/science/article/ pii/S0377221705001736 
[8] G. B. Gelfreikh, Y. A. Nagovitsyn, and E. Y. Nagovitsyna, "Quasi-Periodic Oscillations of Microwave Emission in Solar Active Regions," Publications of the Astronomical Society of Japan, vol. 58, no. 1, pp. 29-35, Feb. 2006. [Online]. Available: http: //pasj.oxfordjournals.org/content/58/1/29

[9] N. E. Huang, Z. Shen, S. R. Long, M. C. Wu, H. H. Shih, Q. Zheng, N.-C. Yen, C. C. Tung, and H. H. Liu, "The empirical mode decomposition and the Hilbert spectrum for nonlinear and nonstationary time series analysis," in Proceedings of the Royal Society of London A: Mathematical, Physical and Engineering Sciences, vol. 454. The Royal Society, 1998, pp. 903-995. [Online]. Available: http: //rspa.royalsocietypublishing.org/content/royprsa/454/1971/903.full.pdf

[10] D. J. Berndt and J. Clifford, "Using Dynamic Time Warping to Find Patterns in Time Series." vol. 10. Seattle, WA, 1994, pp. 359-370, conference Proceedings.

[11] L. Feng, X. Zhao, Y. Liu, Y. Yao, and B. Jin, "A similarity measure of jumping dynamic time warping," vol. 4. IEEE, 2010, pp. 1677-1681, conference Proceedings.

[12] T. Syeda-Mahmood, D. Beymer, and F. Wang, "Shape-based matching of ECG recordings," in Engineering in Medicine and Biology Society, 2007. EMBS 2007. 29th Annual International Conference of the IEEE. IEEE, 2007, pp. 2012-2018. [Online]. Available: http://ieeexplore.ieee.org/xpls/abs_all.jsp?arnumber=4352714

[13] C. A. Ratanamahatana and E. Keogh, "Making time-series classification more accurate using learned constraints." SIAM, 2004, conference Proceedings.

[14] S. L. K. Pond, D. Posada, M. B. Gravenor, C. H. Woelk, and S. D. W. Frost, "GARD: a genetic algorithm for recombination detection," Bioinformatics, vol. 22, no. 24, pp. 3096-3098, Dec. 2006. [Online] Available: http://bioinformatics.oxfordjournals.org/content/22/24/3096

[15] Y.-S. Ding and T.-L. Zhang, "Using Chous pseudo amino acid composition to predict subcellular localization of apoptosis proteins: An approach with immune genetic algorithm-based ensemble classifier," Pattern Recognition Letters, vol. 29, no. 13, pp. 1887-1892, Oct. 2008. [Online]. Available: http://www.sciencedirect.com/science/article/ pii/S0167865508002031

[16] P. Clote, "An efficient algorithm to compute the landscape of locally optimal RNA secondary structures with respect to the Nussinov-Jacobson energy model," Journal of computational biology, vol. 12, no. 1, pp. 83-101, 2005. [Online]. Available: http: //online.liebertpub.com/doi/pdf/10.1089/cmb.2005.12.83

[17] D. J. Wales, J. P. Doye, M. A. Miller, P. N. Mortenson, and T. R. Walsh, "Energy landscapes: from clusters to biomolecules," Advances in Chemical Physics, vol. 115, pp. 1-112, 2000. [Online]. Available: http://www.google.com/books?hl=zh-CN\&lr=\&id=ctG-exL3VocC\&oi= fnd\&pg=PA1\&dq=Wales, + D.+J.,+Doye, + J.+P.+K.+and+Miller,+M. +A.+2000.+Energy+landscapes:+from+clusters+to+biomolecules.+Adv. Chem.Phys\&ots=bZUlB1_sCh\&sig=TshI1fza6yAOzZQxdI4fZ8KeSSg

[18] D. J. Wales, M. A. Miller, and T. R. Walsh, "Archetypal energy landscapes," Nature, vol. 394, no. 6695, pp. 758-760, 1998. [Online]. Available: http://www.nature.com/nature/journal/v394/n6695/ abs/394758a0.html

[19] J. P. Doye, "Network topology of a potential energy landscape: A static scale-free network," Physical review letters, vol. 88, no. 23, p. 238701, 2002. [Online]. Available: http://prl.aps.org/abstract/PRL/v88/ i23/e238701

[20] P. G. Debenedetti and F. H. Stillinger, "Supercooled liquids and the glass transition," Nature, vol. 410, no. 6825, pp. 259-267, 2001. [Online]. Available: http://www.nature.com/nature/journal/v410/n6825/ abs/410259a0.html

[21] A. N. Patel, D. Davis, C. F. Guthrie, D. Tuk, T. T. Nguyen, J. Williams, and others, "Optimizing Cyclic Steam Oil Production with Genetic Algorithms," in SPE Western Regional Meeting. Society of Petroleum Engineers, 2005. [Online]. Available: https: //www.onepetro.org/conference-paper/SPE-93906-MS

[22] C. Xia, P. Guo, T. Shi, and M. Wang, "Speed control of brushless DC motor using genetic algorithm based fuzzy controller," in Proceeding of the 2004 International Conference on Intelligent Mechatronics and Automation, Chengdu, China, 3rd edn. A Treatise on Electricity and Magnetism, vol. 2, 2004, pp. 68-73. [Online]. Available: http://sclab.yonsei.ac.kr/courses/09EC/papers/gaFuzzy2004.pdf

[23] K. L. Shiu and K. Y. Szeto, "Self-adaptive Mutation Only Genetic Algorithm: An Application on the Optimization of Airport Capacity Utilization," in Intelligent Data Engineering and Automated
LearningIDEAL 2008. Springer, 2008, pp. 428-435. [Online]. Available: http://link.springer.com/chapter/10.1007/978-3-540-88906-9 54

[24] C. Messom, "Genetic algorithms for auto-tuning mobile robot motion control," 2002. [Online]. Available: http://mro.massey.ac.nz/handle/ $10179 / 4366$

[25] K. Y. Szeto and L. Y. Fong, "How adaptive agents in stock market perform in the presence of random news: A genetic algorithm approach," in Intelligent Data Engineering and Automated LearningIDEAL 2000. Data Mining, Financial Engineering, and Intelligent Agents. Springer, 2000, pp. 505-510. [Online]. Available: http://link.springer.com/chapter/10.1007/3-540-44491-2_74

[26] L. Y. Fong and K. Y. Szeto, "Rules extraction in short memory time series using genetic algorithms," The European Physical Journal B-Condensed Matter and Complex Systems, vol. 20, no. 4, pp. 569-572, 2001. [Online]. Available: http://link.springer.com/article/10. 1007/PL00011110

[27] M. Benbouziane, "Portfolio selection using genetic algorithm," no. Journal Article, 2012

[28] R. Jiang, K. Y. Szeto, Y. P. Luo, and D. C. Hu, "Distributed parallel genetic algorithm with path splitting scheme for the large traveling salesman problems," in Proceedings of Conference on Intelligent Information Processing, 16th World Computer Congress, 2000, pp. 21-25.

[29] P. Kumar, A. Gupta, V. K. Jayaraman, and B. Kulkarni, "Aligning time series with genetically tuned dynamic time warping algorithm," in Advances in Metaheuristics for Hard Optimization. Springer, 2008, pp. 251-261. [Online]. Available: http://link.springer.com/chapter/10. 1007/978-3-540-72960-0_12

[30] V. Tuzcu and S. Nas, "Dynamic time warping as a novel tool in pattern recognition of ECG changes in heart rhythm disturbances," vol. 1. IEEE, 2005, pp. 182-186, conference Proceedings.

[31] B. Raghavendra, D. Bera, A. S. Bopardikar, and R. Narayanan, "Cardiac arrhythmia detection using dynamic time warping of ECG beats in ehealthcare systems." IEEE, 2011, pp. 1-6, conference Proceedings.

[32] M. Burke and G. Shorten, "Characterisation of ECG profile timing using dynamic time warping," Recent Advances in Telecommunications and Circuit Design, no. Journal Article, pp. 29-34, 2013.

[33] S. Boudaoud, C. Heneghan, H. Rix, O. Meste, and C. O'Brien, "P-wave shape changes observed in the surface electrocardiogram of subjects with obstructive sleep apnoea," in Computers in Cardiology, 2005. IEEE, 2005, pp. 359-362. [Online]. Available: http://ieeexplore.ieee.org/xpls/abs_all.jsp?arnumber=1588111

[34] Y. Ichimaru and G. B. Moody, "Development of the polysomnographic database on CD-ROM," Psychiatry and Clinical Neurosciences, vol. 53, no. 2, pp. 175-177, 1999. [Online]. Available: http: //onlinelibrary.wiley.com/doi/10.1046/j.1440-1819.1999.00527.x/pdf

[35] A. L. Goldberger, L. A. Amaral, L. Glass, J. M. Hausdorff, P. C. Ivanov, R. G. Mark, J. E. Mietus, G. B. Moody, C.-K. Peng, and H. E. Stanley, "Physiobank, physiotoolkit, and physionet components of a new research resource for complex physiologic signals," Circulation, vol. 101, no. 23, pp. e215-e220, 2000. [Online]. Available: http://circ.ahajournals.org/content/101/23/e215.short

[36] W. H. Kruskal and W. A. Wallis, "Use of ranks in onecriterion variance analysis," Journal of the American statistical Association, vol. 47, no. 260, pp. 583-621, 1952. [Online]. Available: http://www.tandfonline.com/doi/abs/10.1080/01621459.1952.10483441

[37] H. B. Mann and D. R. Whitney, "On a test of whether one of two random variables is stochastically larger than the other," The annals of mathematical statistics, pp. 50-60, 1947. [Online]. Available: http://www.jstor.org/stable/2236101

[38] E. S. Lander and D. Botstein, "Mapping mendelian factors underlying quantitative traits using RFLP linkage maps." Genetics, vol. 121, no. 1, pp. 185-199, 1989. [Online]. Available: http://www.genetics.org/ content/121/1/185.short

[39] Y. Zhang and T. F. Edgar, "A robust dynamic time warping algorithm for batch trajectory synchronization." IEEE, 2008, pp. 2864-2869, conference Proceedings.

[40] E. J. Keogh and M. J. Pazzani, "Derivative Dynamic Time Warping." in SDM, vol. 1. SIAM, 2001, pp. 5-7. [Online]. Available: http://epubs.siam.org/doi/pdf/10.1137/1.9781611972719.1 